I was a member of the first Inquiry Steering Group, which encouraged clinicians themselves to notify suicides to the Inquiry. As I understand the approach to be adopted in the next stage, local clinical review for the Inquiry's purpose will inevitably be significantly delayed in a considerable proportion of suicides, as a result of first having to await inquest verdicts in order to establish a full sample of patients. I fear that other problems will then arise, because we have found in Avon that any such delay may severely compromise the collection of full reliable data relevant to clinical audit. Under present clinical conditions, high-risk patients may stay in hospital only very briefly. Unless review takes place quickly, ward staff who had contact with them may find it difficult to furnish detailed reconstructions of what happened. Although the Inquiry will become more effective in identifying a comprehensive sample, it is likely to experience greater difficulty in obtaining full and reliable details from local clinical review. It surely remains important to encourage early clinical audit based on local data collection in advance of inquest verdicts. In Avon we find that our case register of unexpected deaths is able to cover a population of more than half a million easily, reliably and cost-effectively. The Inquiry would indeed be wise both to promote early clinical review and to seek later confirmation following receipt of inquest verdicts.

As clinicians, we can do much to help the Inquiry. The Health of the Nation initiative gives us the opportunity to show that we really can learn valuable lessons from collaborative clinical experience. I fear that unless we make progress on this, the present sharply focused interest in the search for new approaches to suicide prevention will wane, and not return for many a long year. The Inquiry is indeed now at a crucial stage in its development. Surely it is incumbent upon us all to give it our unreserved support, without which it will never achieve its full potential.

Appleby, L., Shaw, J. \& Amos, T. (1997) National Confidential Inquiry into Suicide and Homicide by People with Mental Illness. British journal of Psychiatry. 170, 101-102.

H. G. Morgan Department of Mental Health, University of Bristol, 4I St Michael's Hill, Bristol BS2 $8 D Z$

\section{Measuring cognitive deterioration in Alzheimer's disease}

Sir: Alzheimer's disease (AD) is characterised by progressive cognitive deterioration. We evaluated event-related potential
(ERP) P300 component latency change over 12 months as a biological marker for progression in 22 patients with probable AD.

Baseline and repeat ERP recordings were obtained from 18 women and four men (mean age 70.8 years, s.d. $=5.6$ years, range 58-79) recruited from the memory clinic at St James's Hospital (Swanwick et al, 1996a). P300 latencies were estimated blind to the dates of the recordings. Deterioration in cognition was assessed using the annual rate of change (ARC) for the Mini-Mental State Examination (MMSE), and CAMCOG.

The baseline mean MMSE score was 19.9 (s.d.=3.9); mean CAMCOG score was 64.0 (s.d.=14.1). The mean ARC in MMSE and CAMCOG scores were 2.6 (s.d.=3.9) and 6.5 (s.d.=9.7), respectively. There was a trend for an increase in the P300 latency (paired $t$-test -1.82 , d.f. $=18, P=0.04$ (onetailed)). There was a significant association between the P300 ARC and both the MMSE and CAMCOG ARCs (Kendall Tau $(\tau)=0.39, P<0.01$ and $\tau=0.35, P=0.02$, respectively). However, the magnitude of change on the cognitive scales was significantly greater than for the P300 latency.

The principal findings are that P300 latency increases with dementia severity, and the rate of increase reflects the rate of cognitive decline. The latter finding is consistent with the previously reported finding that, in patients with 'presenile' $\mathrm{AD}$, the degree of cognitive deterioration generally corresponds with P300 latency changes (St Clair et al, 1988).

In a previously reported study of flash visual evoked potentials on the same patients (Swanwick et al, 1996b) no significant increase in latency was demonstrated. This suggests that ERP recordings may provide a more sensitive index of progression in $\mathrm{AD}$.

In conclusion, this study supports the claim by Ball et al (1989) that P300 latency provides a non-invasive, objective marker of change in cognitive function.

Ball, S. S., Marsh, J.T., Schubarth, G., et ol (1989) Longitudinal P300 latency changes in Alzheimer's disease. Journal of Gerontology. 44. MI95-M200.

St Clair, D., Blackburn, I., Blackwood, D., et al (1988) Measuring the course of Alzheimer's disease. A longitudinal study of neuropsychological function and changes in P3 eventrelated potential. British journal of Psychiotry, 152. 48-54.

Swanwick, G. R. J., Coen, R. F., O'Mahony, D., et of (19960) The clinical application of electrophysiological markers in the differential diagnosis of depression and very mild Alzheimer's disease. Journal of Neurology, Neurosurgery \& Psychiotry, 60. 82-86.
_, Rowan, M. J., Coen, R. F., et al (19966) Longitudinal visual evoked potentials in Alzheimer's disease. A preliminary report. Biological Psychiatry, 39, 455-457.

G. R. J. Swanwick 6I Eccles Street, Dublin 7

M. J. Rowan Department of Pharmacology, Trinity College, Dublin

R. F. Coen, B. A. Lawlor, D. Coakley St James's Hospital, Dublin

\section{CYP2D6 genotype and tardive dyskinesia}

Sir: The article by Armstrong et al (1997) on drug-induced movement disorders in schizophrenics in relation to CYP2D6 genotype is important, as research in this area may lead to clues regarding vulnerability to movement disorders such as tardive dyskinesia (TD). The authors conclude that the CYP2D6 genotype may be a factor in determining susceptibility to antipsychotic drug-induced movement disorders including TD. However, this assertion should be treated with caution. Their criteria for diagnosing TD are somewhat arbitrary and, when compared to established criteria such as those by Schooler \& Kane (1982), are liable to diagnose both false positives and false negatives.

We are told that the mutant allele occurs more frequently in the group with chronic movement disorder. This may relate either to TD or to Parkinsonism as both conditions co-existed in the majority of cases. Moreover, this difference between the groups did not reach statistical significance.

The authors have not addressed the issue of masked TD and, in some of their subjects, the movements may have been masked by the neuroleptics. This would have been important to establish in research of this nature.

I feel that the authors should have concluded that the mutant allele predisposed subjects to a chronic movement disorder. Their assertion about TD is premature, not only because of the criteria used, but also because of the mixed clinical picture of their subjects.

Armstrong, M., Daly, A. K., Blennerhasset, R., et ol (1997) Antipsychotic drug-induced movement disorder in schizophrenics in relation to CYP2D6 genotype. British journal of Psychiotry, 170, 23-26.

Schooler, N. C. \& Kane, J.M. (1982) Research diagnoses for tardive dyskinesia. Archives of General Psychiatry, 39, 486-487.

S. H. A. Sajjad St Brigid's Hospital, Ballinasloe,

County Galway, Republic of Ireland 